\title{
Modified Fourth-Order Buck-Boost Converter
}

\author{
FELIX A. HIMMELSTOSS ${ }^{1}$ \& KARL EDELMOSER ${ }^{2}$ \\ ${ }^{1}$ Power Electronics Department \\ University of Applied Science Technikum Wien \\ Hoechstaedtplatz 6, 1200 Wien \\ ${ }^{2}$ Institute of Energy Systems and Electrical Drives \\ University of Technology Vienna \\ Gusshausstraße 24-26, 1040 Wien \\ AUSTRIA \\ ${ }^{1}$ felix.himmelstoss@technikum-wien.at ${ }^{2}$ karl.edelmoser@tuwien.ac.at
}

Abstract: - Some hundred DC/DC converters have been constructed. Here a modified one is investigated. The modification consists in the position of the output capacitor. This does not change the voltage transformation ratio, but leads to constant input current compared to a pulsating one in the original topology. The function of the converter is investigated by inspection and by constructing the signals, the large and small signal model of the converter and the transfer function are derived, dimensioning hints are given and simulations are shown.

Key-Words: - buck-boost converter, constant input current, large signal model, small signal model, simulation Received: June 18, 2019. Revised: December 29, 2019. Accepted: January 25, 2020. Published: February 15, 2020.

\section{Introduction}

There exist many DC/DC topologies to transform DC voltages. They are described in extension in the text books e.g. in $[1,2,3]$. Furthermore, studies were done to construct DC/DC converters $[4,5,6$, 7, 9]. Nevertheless, there are still converters which were not investigated in detail. In this paper we analyze a converter modified from the topology E3 constructed in [6]. The modification is done by a change of the position of the output capacitor. For the three modified basic converters one can consult [8]. The converter (Fig. 1) consists of an active (S) and a passive switch (D), two inductors $\left(\mathrm{L}_{1}, \mathrm{~L}_{2}\right)$ and two capacitors $\left(\mathrm{C}_{1}, \mathrm{C}_{2}\right)$. First we discuss the basics, construct the model of the converter, design the elements and show some simulations.

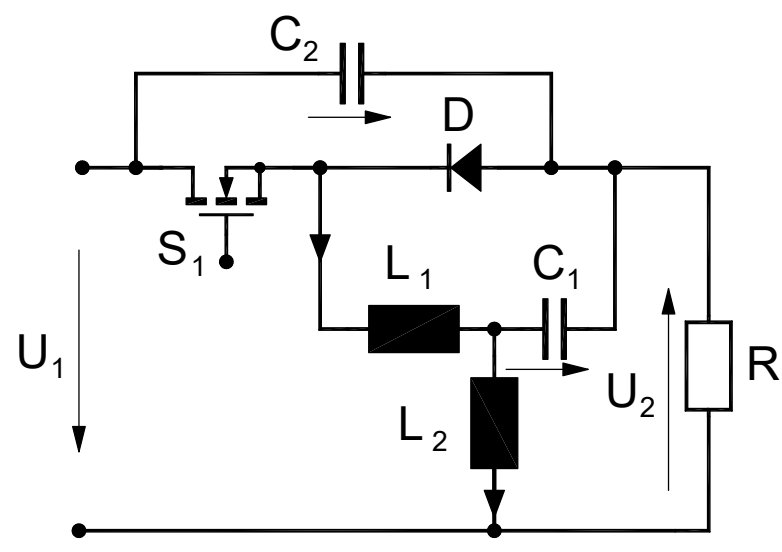

Fig. 1. Modified buck-boost converter

\section{Basic investigations}

\subsection{Voltage transformation ratio}

The directions of the currents and the voltages are taken as they are. These directions can be found by simple inspections. For the basic analyzes we assume ideal devices, that means no parasitic resistors in the devices and infinitesimal switching speed of the semiconductors. The converter is already in the steady state mode (the signals in each period look the same), and is in the continuous inductor current mode (the inductor currents are positive during the whole switching period). The capacitors are chosen so large, that the voltages across them are nearly constant during the switching period.

Due to the fact that in steady state the voltages across the inductors have to be zero in the mean, one gets

$$
\begin{aligned}
& U_{C 2}=U_{1}+U_{2} \\
& U_{C 1}=U_{2} .
\end{aligned}
$$

Another interesting aspect is that the voltage across $\mathrm{L}_{2}$ is always the same in both modes

$$
u_{L 2}=u_{C 1}-u_{C 2}+u_{1} \text {. }
$$

The voltages across the capacitors almost do not change during one switching period (because of appropriate large capacitor values). Therefore, the voltage must be practically always zero and the current through $\mathrm{L}_{2}$ must be constant. 
The voltage transformation ratio can be calculated from the voltage-time balance of inductor $\mathrm{L}_{1}$ only. One can write

$$
\begin{aligned}
& \left(U_{C 2}-U_{C 1}\right) \cdot d=\left|-U_{C 1}\right| \cdot(1-d) \\
& U_{C 2} \cdot d=U_{C 1}=U_{2} .
\end{aligned}
$$

Therefore, one gets for the output voltage

$$
U_{2}=\frac{d}{1-d} U_{1} .
$$

A sketch of the voltages across the coils is shown in Fig. 2. The control signal is high during one third of the switching period. (a)

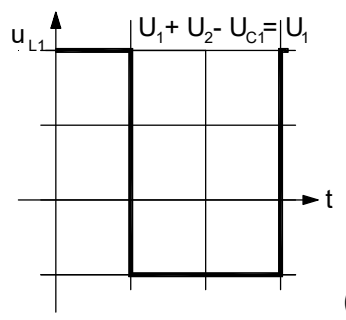

(b)

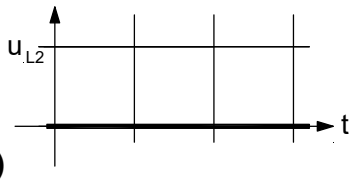

Fig. 2. Voltage across the inductors (a) $\mathrm{L}_{1}$, (b) $\mathrm{L}_{2}$

\subsection{Voltage stress across the semiconductors}

For the design of the converter the voltage stress across the active and the passive switches (Fig. 3 ) is very important. With KVL one gets for the voltage across the transistor during mode M1 the sum of the input and the output voltages

$$
u_{S}=U_{1}+U_{2} \text {. }
$$

The same, but negative voltage can be found across the diode during mode M1

$$
u_{D}=-\left(U_{1}+U_{2}\right) .
$$

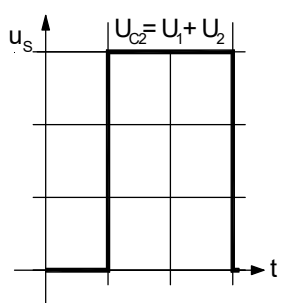

(b)

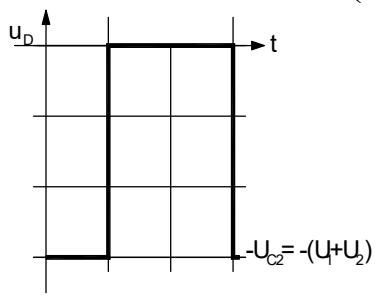

Fig. 3. Voltage across the semiconductors (a) switch, (b) diode

\subsection{Connection between the currents}

For the design also the amount of currents through the elements is important. We start from the output current (Fig. 4). The load current is nearly constant, because of the almost constant output voltage.

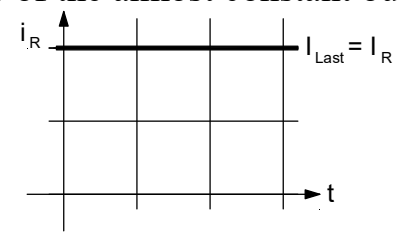

Fig. 4. Load current
The current through $\mathrm{C}_{1}$ is the same during both modes, i.e. always the difference of the currents through the inductors

$$
i_{C 1}=i_{L 1}-i_{L 2} \text {. }
$$

The mean value of the capacitor current is zero in the steady state. Therefore, one can deduce that the mean values of the inductor currents must be the same

$$
\bar{I}_{L 1}=\bar{I}_{L 2} .
$$

Further information one gets from the charge balance of $\mathrm{C}_{2}$. During M1 one gets from KCL

$$
i_{C 2}=-\left(i_{L 1}-i_{L 2}\right)-I_{R}
$$

and during $\mathrm{M} 2$

$$
i_{C 2}=-I_{R}+\bar{I}_{2} .
$$

A sketch of the current through $\mathrm{C}_{2}$ is shown in Fig. 5.a.

(a)

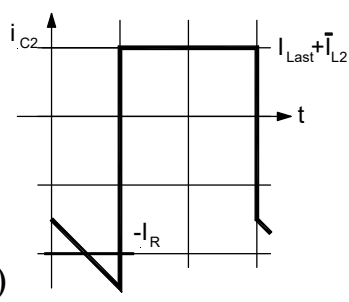

(b)

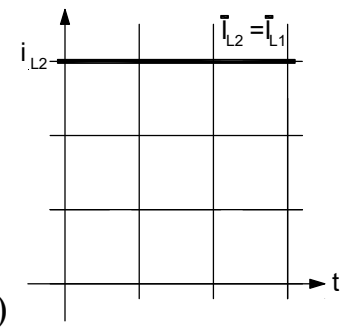

Fig. 5. Current through (a) $\mathrm{C}_{2}$ and $\mathrm{L}_{2}$ (b)

Now it is easy to sketch the current through $\mathrm{L}_{2}$ (Fig. 5.b). The current through $\mathrm{L}_{1}$ (Fig. 6.a) has the same mean value, but has a trapezoid form. (a)

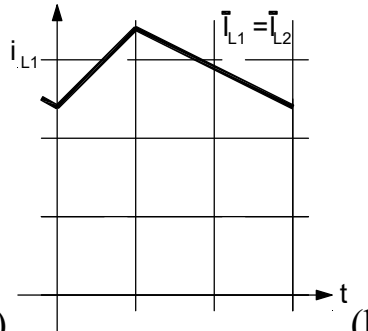

(b)

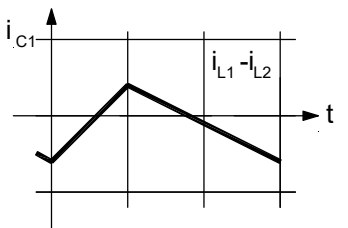

Fig. 6. Current through (a) $\mathrm{L}_{1}$ and (b) through $\mathrm{C}_{1}$

The current through $\mathrm{C}_{1}$ (Fig. 6.b) results from the ripple through $\mathrm{L}_{1}$. From the charge-balance of $\mathrm{C}_{2}$ one gets for the mean values of the inductor currents in dependence on the load current and the duty cycle

$$
\begin{aligned}
& I_{\text {Load }} d=\left(-I_{\text {Load }}+\bar{I}_{L 2}\right)(1-d) \\
& \bar{I}_{L 1}=\bar{I}_{L 2}=\frac{1}{1-d} I_{\text {Load }} .
\end{aligned}
$$

The current depends on the duty cycle. From (6) one can calculate the duty cycle depending on the input and output voltages according to

$$
\begin{aligned}
& U_{2}-d \cdot U_{2}=d \cdot U_{1} \\
& d=\frac{U_{2}}{U_{1}+U_{2}} .
\end{aligned}
$$


With

$$
1-d=1-\frac{U_{2}}{U_{1}+U_{2}}=\frac{U_{1}}{U_{1}+U_{2}}
$$

the mean values of the inductor currents depending on the load current, the input and the output voltages can be written according to

$$
\bar{I}_{L 1}=\bar{I}_{L 2}=\frac{U_{1}+U_{2}}{U_{1}} I_{\text {Load }} .
$$

Only the current through the inductor $\mathrm{L}_{1}$ is flowing through the semiconductors when they are turned on

(a)

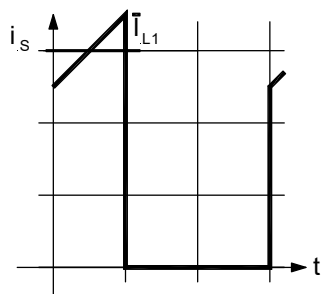

(b)

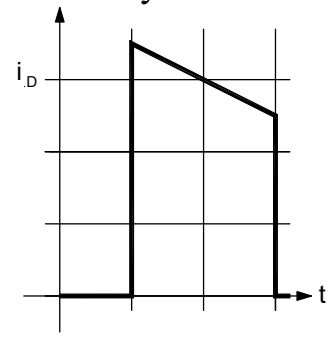

Fig. 7. Current through the semiconductors (a) switch, (b) diode

One very interesting point of this converter is the constant input current (Fig. 8).

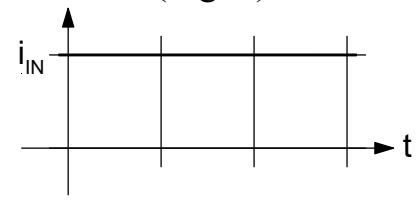

Fig. 8. Input current of the converter

\section{Modelling}

\subsection{Idealized converter}

The state equations for the idealized converter in mode M1 (active switch is conducting, passive switch blocks) are

$$
\begin{aligned}
& \frac{d i_{L 1}}{d t}=\frac{u_{C 2}-u_{C 1}}{L_{1}} \\
& \frac{d i_{L 2}}{d t}=\frac{u_{C 1}-u_{C 2}+u_{1}}{L_{2}} \\
& \frac{d u_{C 1}}{d t}=\frac{i_{L 1}-i_{L 2}}{C_{1}} \\
& \frac{d u_{C 2}}{d t}=\frac{-i_{L 1}+i_{L 2}-\left(-u_{1}+u_{C 2}\right) / R}{C_{2}} .
\end{aligned}
$$

The state equations for the idealized converter in mode M2 (active switch blocks, passive switch is conducting) are

$$
\begin{aligned}
\frac{d i_{L 1}}{d t} & =\frac{-u_{C 1}}{L_{1}} \\
\frac{d i_{L 2}}{d t} & =\frac{u_{C 1}-u_{C 2}+u_{1}}{L_{2}}
\end{aligned}
$$

$$
\begin{aligned}
\frac{d u_{C 1}}{d t} & =\frac{i_{L 1}-i_{L 2}}{C_{1}} \\
\frac{d u_{C 2}}{d t} & =\frac{i_{L 2}-\left(-u_{1}+u_{C 2}\right) / R}{C_{2}}
\end{aligned}
$$

The general state-space description consists of the state-equation (27) and the output equation (28)

$$
\begin{aligned}
& \frac{d}{d t} \underline{x}=A \underline{x}+B \underline{u} \\
& y=C \underline{x}+D \underline{u} .
\end{aligned}
$$

In the continuous inductor current mode, the converter has only two stages. The matrixes are marked with a line in mode M1 and with two lines above the symbols in mode M2. When the time constants of the system are large compared to the switching period, both equations can be combined by weighting them with the time they are valid within one period. To obtain the large system model, the two weighted model descriptions are added and the sum is divided by the period.

$$
\left.\begin{array}{l}
\frac{d}{d t} \underline{x}=\bar{A} \underline{x}+\bar{B} \underline{u} \mid \cdot d \\
\frac{d}{d t} \underline{x}=\bar{A} \underline{x}+\bar{B} \underline{u} \mid \cdot(1-d)
\end{array}\right\}+
$$

The state-matrix for the two modes is

$$
\bar{A}=\left[\begin{array}{cccc}
0 & 0 & -\frac{1}{L_{1}} & \frac{1}{L_{1}} \\
0 & 0 & \frac{1}{L_{2}} & -\frac{1}{L_{2}} \\
\frac{1}{C_{1}} & -\frac{1}{C_{1}} & 0 & 0 \\
-\frac{1}{C_{2}} & \frac{1}{C_{2}} & 0 & -\frac{1}{C_{2} R}
\end{array}\right]
$$

and is

$$
A=\left[\begin{array}{cccc}
0 & 0 & -\frac{1}{L_{1}} & 0 \\
0 & 0 & \frac{1}{L_{2}} & -\frac{1}{L_{2}} \\
\frac{1}{C_{1}} & -\frac{1}{C_{1}} & 0 & 0 \\
0 & \frac{1}{C_{2}} & 0 & -\frac{1}{C_{2} R}
\end{array}\right] .
$$

The state matrix for the large signal model is therefore

$$
A=\left[\begin{array}{cccc}
0 & 0 & -\frac{1}{L_{1}} & \frac{d}{L_{1}} \\
0 & 0 & \frac{1}{L_{2}} & -\frac{1}{L_{2}} \\
\frac{1}{C_{1}} & -\frac{1}{C_{1}} & 0 & 0 \\
-\frac{d}{C_{2}} & \frac{1}{C_{2}} & 0 & -\frac{1}{C_{2} R}
\end{array}\right] .
$$

The input matrix can be written according to 


$$
\bar{B}=\bar{B}=\left[\begin{array}{c}
0 \\
\frac{1}{L_{2}} \\
0 \\
1 \\
\hline C_{2} R
\end{array}\right]
$$

leading to the state-equation

$$
\frac{d}{d t}\left(\begin{array}{c}
i_{L 1} \\
i_{L 2} \\
u_{C 1} \\
u_{C 2}
\end{array}\right)=\left[\begin{array}{cccc}
0 & 0 & -\frac{1}{L_{1}} & \frac{d}{L_{1}} \\
0 & 0 & \frac{1}{L_{2}} & -\frac{1}{L_{2}} \\
\frac{1}{C_{1}} & -\frac{1}{C_{1}} & 0 & 0 \\
-\frac{d}{C_{2}} & \frac{1}{C_{2}} & 0 & -\frac{1}{C_{2} R}
\end{array}\right]\left(\begin{array}{c}
i_{L 1} \\
i_{L 2} \\
u_{C 1} \\
u_{C 2}
\end{array}\right)+\left[\begin{array}{c}
0 \\
\frac{1}{L_{2}} \\
0 \\
\frac{1}{C_{2} R}
\end{array}\right]\left(u_{1}\right)^{\prime}
$$

The output equation can be obtained by

$$
y=u_{2}=\left[\begin{array}{llll}
0 & 0 & 0 & 1
\end{array}\right]\left(\begin{array}{c}
i_{L 1} \\
i_{L 2} \\
u_{C 1} \\
u_{C 2}
\end{array}\right)+[-1]\left(u_{1}\right)
$$

\subsection{Linearized idealized converter}

The state equation is a non-linear one, caused by the multiplication of two variables $\left(d \cdot u_{C 2}, d \cdot i_{L 1}\right)$. To obtain a transfer function of the converter, the system must be linearized. This is done by the perturbation method. The variables are written as the value of the working point (written with capital letters and a zero in the index) added with a small perturbation (written with small letters with roof on top)

$$
\begin{array}{lll}
i_{L 1}=I_{L 10}+\hat{i}_{L 1} & i_{L 2}=I_{L 20}+\hat{i}_{L 2} & u_{C 1}=U_{C 10}+\hat{u}_{C 1} \\
u_{C 2}=U_{C 20}+\hat{u}_{C 2} & u_{1}=U_{10}+\hat{u}_{1} & d=D_{0}+\hat{d}
\end{array}
$$

The linearization is shown for the first equation

$$
\frac{d i_{L 1}}{d t}=-\frac{1}{L_{1}} u_{C 1}+\frac{d}{L_{1}} u_{C 2} \text {. }
$$

Inserting the perturbed values leads to

$$
\frac{d i_{L 1}}{d t}=0+\frac{d \hat{i}_{L 1}}{d t}=\frac{1}{L_{1}}\left\{-\left(U_{C 10}+\hat{u}_{C 1}\right)+\left(D_{0}+\hat{d}\right)\left(U_{C 20}+\hat{u}_{C 2}\right)\right\} \cdot
$$

The multiplication leads to terms which consist of working point values, terms which are products of a working point value with a disturbance (a small signal value) and products of two small signal values. This equation is linearized when the product of the two perturbations is deleted. This leads to the small signal equation of the derivative of the current through $\mathrm{L}_{1}$ according to

$$
\frac{d \hat{i}_{L 1}}{d t}=\frac{1}{L_{1}}\left\{-\hat{u}_{C 1}+D_{0} \hat{u}_{C 2}+U_{C 20} \hat{d}\right\} .
$$

For the working point one gets the connection leading to

$$
0=-U_{C 10}+D_{0} U_{C 20}
$$

$$
U_{C 20}=\frac{1}{D_{0}} U_{C 10} .
$$

Using the disturbance concept for all four stateequations results in the state matrix

$$
A_{L}=\left[\begin{array}{cccc}
0 & 0 & -\frac{1}{L_{1}} & \frac{D_{0}}{L_{1}} \\
0 & 0 & \frac{1}{L_{2}} & -\frac{1}{L_{2}} \\
\frac{1}{C_{1}} & -\frac{1}{C_{1}} & 0 & 0 \\
-\frac{D_{0}}{C_{2}} & \frac{1}{C_{2}} & 0 & -\frac{1}{C_{2} R}
\end{array}\right]
$$

and in the input matrix

$$
B_{L}=\left[\begin{array}{cc}
0 & \frac{U_{C 20}}{L_{1}} \\
\frac{1}{L_{2}} & 0 \\
0 & 0 \\
\frac{1}{R C_{2}} & -\frac{I_{L 10}}{C_{2}}
\end{array}\right]
$$

The linearized model has now the duty cycle $d$ as an additional input variable. The input vector must now be written as

$$
\underline{u}=\left(\begin{array}{c}
\hat{u_{1}} \\
\hat{d}
\end{array}\right) .
$$

The idealized small signal model is therefore

$$
\frac{d}{d t}\left(\begin{array}{l}
\hat{i}_{L 1} \\
\hat{i}_{L 2} \\
\hat{u}_{C 1} \\
\hat{u}_{C 2}
\end{array}\right)=\left[\begin{array}{cccc}
0 & 0 & -\frac{1}{L_{1}} & \frac{D_{0}}{L_{1}} \\
0 & 0 & \frac{1}{L_{2}} & -\frac{1}{L_{2}} \\
\frac{1}{C_{1}} & -\frac{1}{C_{1}} & 0 & 0 \\
-\frac{D_{0}}{C_{2}} & \frac{1}{C_{2}} & 0 & -\frac{1}{C_{2} R}
\end{array}\right]\left(\begin{array}{l}
\hat{i}_{L 1} \\
\hat{i}_{L 2} \\
\hat{u}_{C 1} \\
\hat{u}_{C 2}
\end{array}\right)+\left[\begin{array}{cc}
0 & \frac{U_{C 20}}{L_{1}} \\
\frac{1}{L_{2}} & 0 \\
0 & 0 \\
\frac{1}{C_{2} R} & -\frac{I_{L 10}}{C_{2}}
\end{array}\right]\left(\begin{array}{l}
\hat{u}_{1} \\
d
\end{array}\right)
$$

\subsection{Calculation of the transfer function}

The state equation in matrix form is now written with abbreviations as

$$
\frac{d}{d t}\left(\begin{array}{l}
\hat{i}_{L 1} \\
\hat{i}_{L 2} \\
\hat{u}_{C 1} \\
\hat{u}_{C 2}
\end{array}\right)=\left[\begin{array}{cccc}
0 & 0 & A_{13} & A_{14} \\
0 & 0 & A_{23} & A_{24} \\
A_{31} & A_{32} & 0 & 0 \\
A_{41} & A_{42} & 0 & A_{44}
\end{array}\right]\left(\begin{array}{l}
\hat{i}_{L 1} \\
\hat{i}_{L 2} \\
\hat{u}_{C 1} \\
\hat{u}_{C 2}
\end{array}\right)+\left[\begin{array}{cc}
0 & B_{12} \\
B_{21} & 0 \\
0 & 0 \\
B_{41} & B_{42}
\end{array}\right]\left(\begin{array}{l}
\hat{u}_{1} \\
d
\end{array}\right) .
$$

Laplace transformation leads to

$$
\left[\begin{array}{cccc}
s & 0 & 0 & 0 \\
0 & s & 0 & 0 \\
0 & 0 & s & 0 \\
0 & 0 & 0 & s
\end{array}\right]\left(\begin{array}{c}
I_{L 1}(s) \\
I_{L 2}(s) \\
U_{C 1}(s) \\
U_{C 2}(s)
\end{array}\right)=\left[\begin{array}{cccc}
0 & 0 & A_{13} & A_{14} \\
0 & 0 & A_{23} & A_{24} \\
A_{31} & A_{32} & 0 & 0 \\
A_{41} & A_{42} & 0 & A_{44}
\end{array}\right]\left(\begin{array}{c}
I_{L 1}(s) \\
I_{L 2}(s) \\
U_{C 1}(s) \\
U_{C 2}(s)
\end{array}\right)+\left[\begin{array}{cc}
0 & B_{12} \\
B_{21} & 0 \\
0 & 0 \\
B_{41} & B_{42}
\end{array}\right]\left(\begin{array}{l}
U_{1}(s) \\
D(s)
\end{array}\right)
$$

Written as a fourth order equation system gives 


$$
\left[\begin{array}{cccc}
s & 0 & -A_{13} & -A_{14} \\
0 & s & -A_{23} & -A_{24} \\
-A_{31} & -A_{32} & s & 0 \\
-A_{41} & A_{42} & 0 & s-A_{44}
\end{array}\right]\left(\begin{array}{c}
I_{L 1}(s) \\
I_{L 2}(s) \\
U_{C 1}(s) \\
U_{C 2}(s)
\end{array}\right)=\left[\begin{array}{cc}
0 & B_{12} \\
B_{21} & 0 \\
0 & 0 \\
B_{41} & B_{42}
\end{array}\right]\left(\begin{array}{l}
U_{1}(s) \\
D(s)
\end{array}\right)
$$

The most important transfer function is the connection between the voltage across $\mathrm{C}_{2}$ and the duty cycle $\mathrm{d}$. The numerator and the denominator can be calculated according Crammer's rule and with the help of the developing axiom leading to

$$
\begin{aligned}
\frac{U_{C 2}(s)}{D(s)} & =-\frac{N_{-} U_{C 2} D}{D} \\
\frac{U_{C 2}(s)}{U_{1}(s)} & =-\frac{N_{-} U_{C 2} U_{1}}{D}
\end{aligned}
$$

With the abbreviations $(51,52,53)$

$$
\begin{aligned}
& N_{-} U_{C 2} D=\frac{I_{L 10}}{C_{2}} s^{3}+\frac{D_{0} U_{C 20}}{C_{2} L_{1}} s^{2}+\frac{I_{L 10}\left(L_{1}+L_{2}\right)}{C_{1} C_{2} L_{1} L_{2}} s-\frac{1-D_{0}}{C_{1} C_{2} L_{2}} \frac{U_{C 20}}{L_{1}} \\
& N_{-} U_{C 2} U_{1}=s\left[s^{2} \frac{I_{L 10}}{C_{2}}+s \frac{D_{0} U_{C 20}}{C_{2} L_{1}}+\frac{I_{L 10}\left(L_{1}+L_{2}\right)}{C_{1} C_{2} L_{1} L_{2}}\right] \\
& D=s^{4}+s^{3}\left(\frac{1}{C_{2} R}\right)+s^{2}\left(\frac{D_{0}^{2}}{C_{2} L_{1}}+\frac{1}{C_{2} L_{2}}+\frac{L_{1}+L_{2}}{C_{1} L_{1} L_{2}}\right)+s\left(\frac{L_{1}+L_{2}}{C_{1} C_{2} L_{1} L_{2} R}\right)+\left(\frac{\left(1-D_{0}\right)^{2}}{C_{1} C_{2} L_{1} L_{2}}\right)
\end{aligned}
$$

\subsection{Modeling with parasitic resistors}

The equivalent circuits for the two modes are shown in Figs. 9.a and 9.b. The results are shown in the appendix (A.1, A.2).

(a)

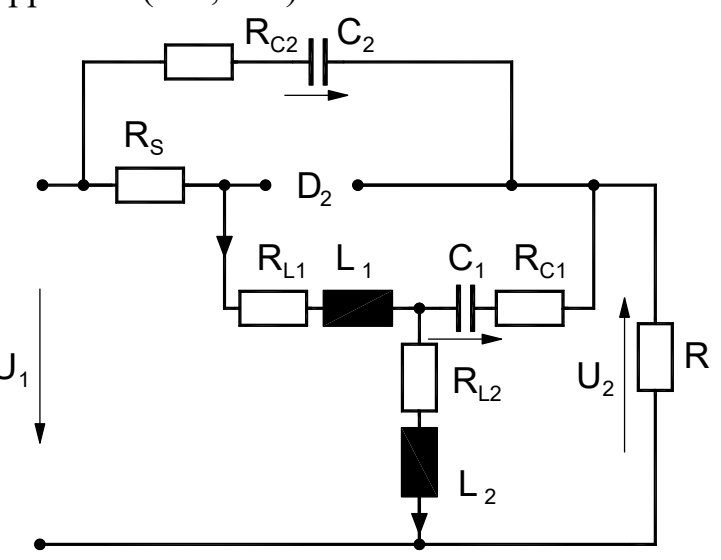

(b)

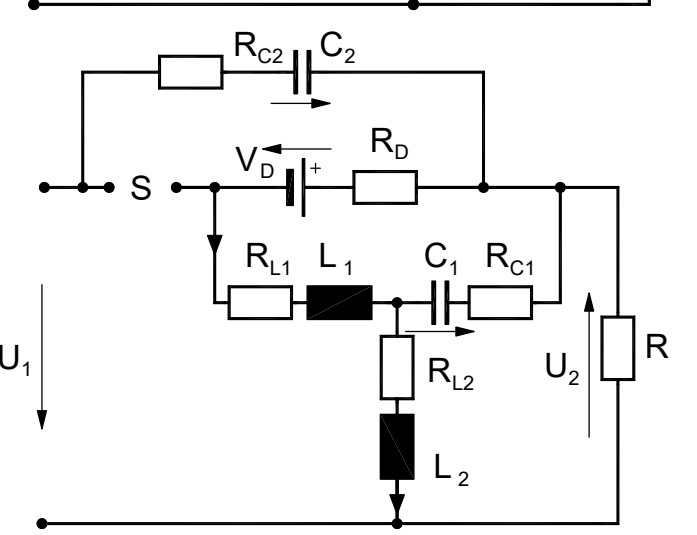

Fig. 9. Equivalent circuit of mode (a) M1 and (b) M2
We model now the bidirectional converter (Fig. 11). Both semiconductor switches have the same on-resistor value.

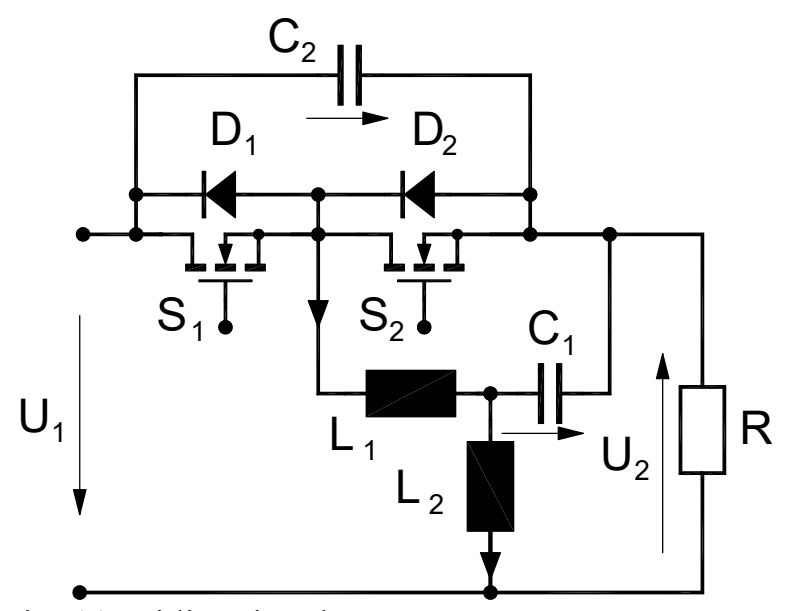

Fig. 11. Bidirectional converter

The large signal model (A3) and the small signal model (A4) are written in the appendix. The small signal model can be derived similar to the process shown in 2.2 .

\section{Dimensioning hints}

The voltage and current stress of the devices can be taken from part 2. During the on-time of the active switch $\mathrm{C}_{2}$ is discharged by the load current. The voltage changes by

$$
\Delta u_{C 2}=\frac{1}{C_{2}} \int_{0}^{d T} I_{\text {Load }} d t .
$$

Solving, including (16) and rearranging leads to

$$
C_{2}=\frac{1}{\Delta u_{C 2}} \cdot \frac{I_{\text {Load }} U_{2}}{f\left(U_{1}+U_{2}\right)} .
$$

The equivalent series resistor of the capacitor causes a higher capacitor value. The input voltage is across the inductor $\mathrm{L}_{1}$ and the current increases during the on-time $d T$ by $\Delta I_{L}$. Including (16) and rearranging gives

$$
L_{1}=\frac{U_{1} U_{2}}{\left(U_{1}+U_{2}\right) f \Delta I_{L}} .
$$

With a voltage ripple across $\mathrm{C}_{1}$ of $\Delta \mathrm{u}_{\mathrm{C} 1}$ and graphical integration

$$
\Delta u_{C 1}=\frac{1}{C_{1}} \int_{\frac{d T}{2}}^{T-\frac{(1-d) T}{2}} i_{C 1}^{2} d t=\frac{1}{C_{1}}\left[\frac{\Delta I_{2}}{2} \frac{d T}{2} \frac{1}{2}+\frac{\Delta I_{2}}{2} \frac{(1-d) T}{2} \frac{1}{2}\right]
$$

one gets

$$
C_{1}=\frac{\Delta I_{L}}{8 f \Delta u_{C 1}} .
$$

The resonance frequency $f_{R}$ of the tank $L_{2}$ and $C_{1}$ can be chosen, e.g. one tenth of the switching 
frequency, to achieve a good damping of the switching noise. This leads to a value for $\mathrm{L}_{2}$ according to

$$
L_{2}=\frac{1}{4 \pi^{2} f_{R}^{2} C_{1}} .
$$

\section{Simulations}

\subsection{Steady state}

In Figs. 12 and 13 the voltage across and the current through the diode, the transistor, and the inductors are shown. In the last diagram one can see the input voltage, the control signal and the output voltage. The duty cycle was chosen to about one third as in the sketches in part 2. In Fig. 14 the currents through capacitors and the input and output currents are depicted.

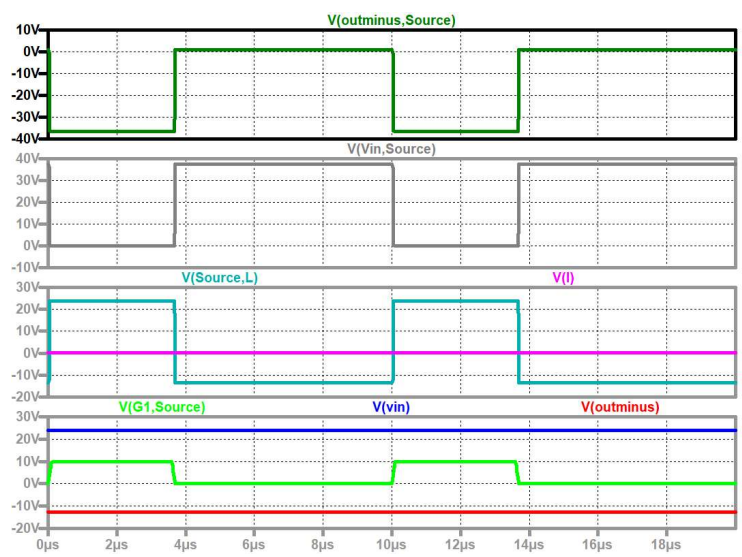

Fig. 12. Up to down: voltage across the diode, voltage across the active switch, voltage across the inductors; input voltage, control signal, output voltage

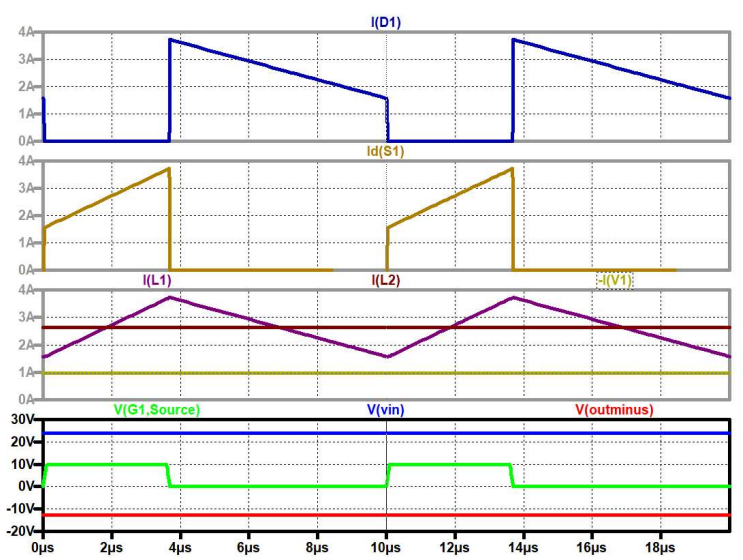

Fig. 13. Up to down: current through the diode, current through the active switch, current through the inductors; input voltage, control signal, output voltage

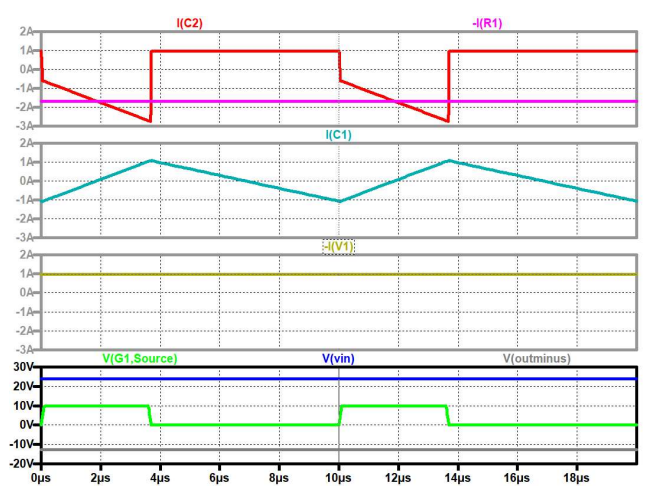

Fig. 14. Up to down: current through $\mathrm{C}_{2}$ and load current, current through $\mathrm{C}_{1}$, input current; input voltage, control signal, output voltage

\subsection{Dynamic behavior}

In the Figs. 15 to 19 one can see the behavior of the circuit when the input voltage is turned on (Fig. 15), during the soft start (Fig. 16), during an input voltage step (Fig. 17), after a load step (Fig. 18), and when the duty cycle ramps down (Fig. 19). The signals are up to down: the load current, the input and output voltages, the current through the inductors, and the control signal of the duty cycle (the input signal of the pwm modulator).

When the input voltage is applied (Fig. 15), a short ringing occurs, because the capacitors and the inductors form resonance circuits. These ringings vanish within $10 \mathrm{~ms}$. Fig. 16 shows the soft-start of the converter. The control signal ramps until a duty cycle of $50 \%$ is reached. When the preset end value is reached, a small ringing occurs. Fig. 17 shows the influence of an input voltage step. This causes a large ringing, especially in the inductor $\mathrm{L}_{1}$. In Fig. 18 the influence of a load step is shown. This leads again in a pronounced ringing. In Fig. 19 the duty cycle is reduced by a ramp. The converter values are $\mathrm{L}_{1}=47 \mu \mathrm{H}, \mathrm{L}_{2}=5 \mu \mathrm{H}, \mathrm{C}_{1}=\mathrm{C}_{2}=330 \mu \mathrm{F}$.

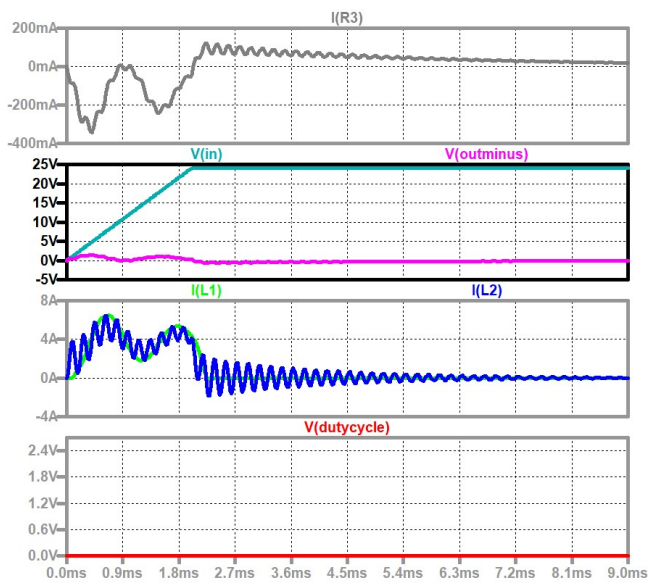

Fig. 15. Power on (signals as in Fig. 16) 


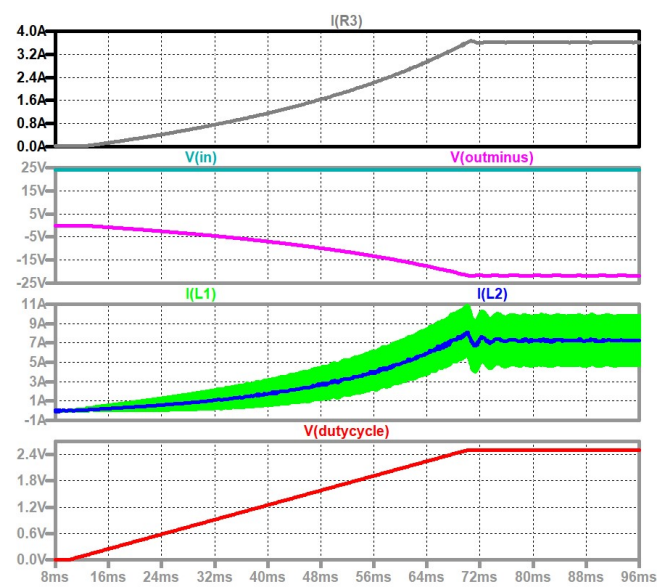

Fig. 16. Soft-start, up to down: current through the load; input voltage, output voltage; current through the inductors; duty cycle control signal

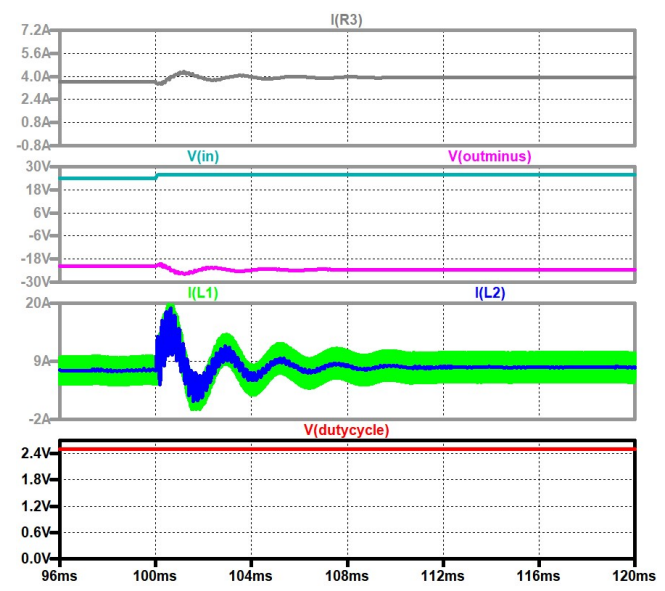

Fig. 17. Input voltage step, up to down: current through the load; input voltage, output voltage; current through the inductors; duty cycle control signal

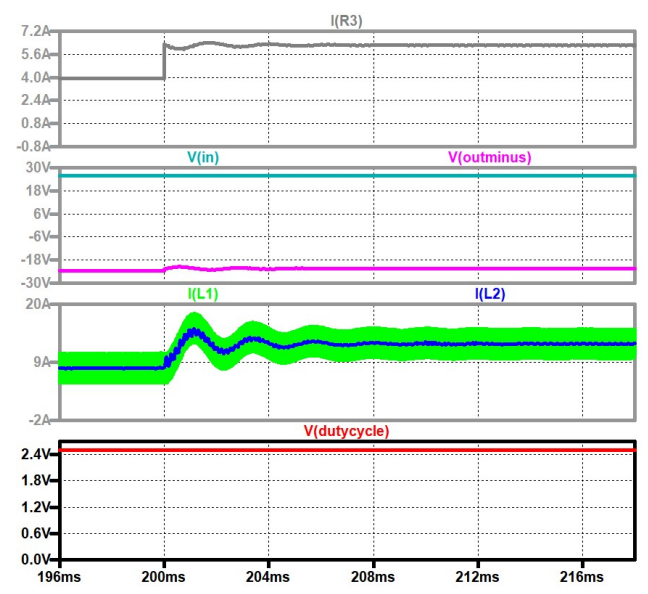

Fig. 18. Load current step, up to down: current through the load; input voltage, output voltage; current through the inductors; duty cycle control signal

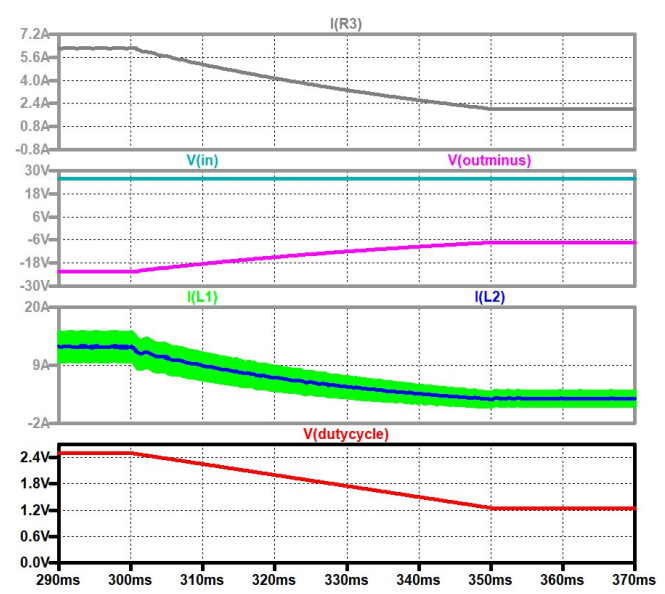

Fig. 19. Duty cycle ramp down, up to down: current through the load; input voltage, output voltage; current through the inductors; duty cycle control signal

Some additional notes to Fig. 18. A step of the input voltage leads to an increased output voltage and therefore to an increased load current. The converter consists of resonant circuits and this causes ringing, especially pronounced in the inductor currents. In this case the duty cycle of the converter is constant, only the input voltage makes a step. The input voltage should change only slowly. This is commendable for all modified converters where the bulk capacitor is connected between the input and the output.

The voltage across the bulk capacitor is the sum of the input and output voltages, so more energy is stored compared to a converter where the bulk capacitor is between the output connectors, when the same capacitor value is used, or a smaller capacitor value can be used for the same stored energy. The larger the bulk capacitor the lower the frequency of the ringing in case of fast changes of the load, the input voltage or the duty cycle. The duty cycle should always change with a ramp as shown during soft-start (Fig. 16) and when the duty cycle has to be altered (Fig. 19).

It should be mentioned that this converter can also be used (in the two-quadrant version) in battery or super-capacitor energy-storage systems and especially in the one-quadrant version for application in lighting devices. In these cases the output current has to be controlled.

\section{Conclusions}

The paper was written in a more didactical way, especially in the chapters 2 and 3. Main aspects of this new buck-boost converter are 
- Constant input and output current

- Capacitor $\mathrm{C}_{1}$ and inductor $\mathrm{L}_{2}$ form a lowpass filter

- Voltage and current stress are equal to those of the normal buck-boost converter

- The voltage across the bulk capacitor is the sum of the input and output voltages

The low-pass filter suppresses the switching noise and increases the electromagnetic compatibility. The converter can be used, because of its buck-boost behavior, where step-up, and step-down of the input voltage and a large voltage transformation ratio are necessary, e.g. in auxiliary drives with DC motors in automotive applications. Other loads, especially when the output current is controlled, are lighting applications, especially for LEDs.

\section{References:}

[1] N. Mohan, T. Undeland and W. Robbins, Power Electronics, Converters, Applications and Design, 3nd ed. New York: W. P. John Wiley \& Sons, 2003.

[2] F. Zach, Power Electronics, in German: Leistungselektronik, Wien: Springer, 6th ed., 2020.
[3] Y. Rozanov, S. Ryvkin, E. Chaplygin, P. Voronin, Power Electronics Basics, CRC Press, 2016.

[4] S. Cuk, General topological properties of switching structures, IEEE Power Electronics Specialists Conference, San Diego, CA, USA, 1979, pp. 109-130.

[5] D. Maksimovic and S. Cuk, Switching converters with wide DC conversion range, IEEE Transactions on Power Electronics, Vol. 6, No. 1, pp. 151-157, Jan. 1991.

[6] B. W. Williams, Generation and Analysis of Canonical Switching Cell DC-to-DC Converters, IEEE Transactions on Industrial Electronics, Vol. 61, No. 1, pp. 329-346, Jan. 2014.

[7] R. Marquez and M. A. Contreras-Ordaz, The ThreeTerminal Converter Cell, Graphs, and Generation of DCto-DC Converter Families, IEEE Transactions on Power Electronics, Vol. 35, No. 8, pp. 7725-7728, Aug. 2020

[8] F. A. Himmelstoss, and K. H. Edelmoser, Modified Basic DC-DC Converters, Power Conversion and Intelligent Motion PCIM 2018, pp. 1076-1083.

[9] F. A. Himmelstoss and H. L. Votzi, A family of quadratic DC/DC converters with one low-side switch and a tapped inductor at the output side, 2019 International Aegean Conference on Electrical Machines and Power Electronics (ACEMP) \& 2019 International Conference on Optimization of Electrical and Electronic Equipment (OPTIM), Istanbul, Turkey, 2019, pp. 304-309

\section{APPENDIX}

A.1. Bidirectional converter - weighted model (large signal model)

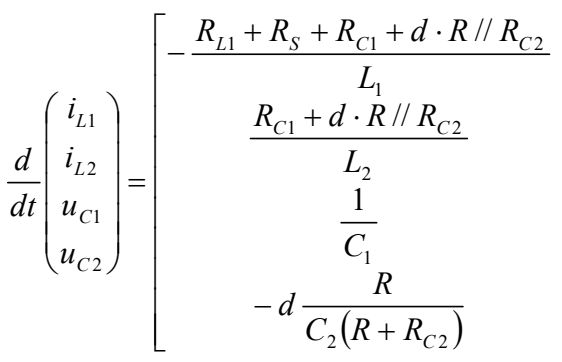

$$
\begin{gathered}
\frac{R_{C 1}+d \cdot R / / R_{C 2}}{L_{1}} \\
-\frac{1}{L_{2}}\left[R_{L 2}+d \cdot R_{C 1}+R / / R_{C 2}\right] \\
-\frac{1}{C_{1}} \\
\frac{1}{C_{2}} \frac{R}{R+R_{C 2}}
\end{gathered}
$$$$
\left.\begin{array}{cc}
-\frac{1}{L_{1}} & \frac{d R}{L_{1}\left(R+R_{C 2}\right)} \\
\frac{1}{L_{2}} & -\frac{1}{L_{2}} \frac{R}{R+R_{C 2}} \\
0 & 0 \\
0 & -\frac{1}{C_{2}\left(R+R_{C 2}\right)}
\end{array}\right]\left(\begin{array}{c}
i_{L 1} \\
i_{L 2} \\
u_{C 1} \\
u_{C 2}
\end{array}\right)+\left[\begin{array}{c}
0 \\
\frac{1}{L_{2}} \frac{R}{R_{S}+R_{C 2}} \\
0 \\
\frac{1}{C_{2}\left(R+R_{C 2}\right)}
\end{array}\right]\left(u_{1}\right)(A
$$

A.2. Bidirectional converter - linearized model (small signal model)

$$
\begin{aligned}
& A=\left[\begin{array}{cccc}
-\frac{R_{L 1}+R_{S}+R_{C 1}+D_{0} \cdot R / / R_{C 2}}{L_{1}} & \frac{R_{C 1}+D_{0} \cdot R / / R_{C 2}}{L_{1}} & -\frac{1}{L_{1}} & \frac{D_{0} R}{L_{1}\left(R+R_{C 2}\right)} \\
\frac{R_{C 1}+D_{0} \cdot R / / R_{C 2}}{L_{2}} & -\frac{1}{L_{2}}\left[R_{L 2}+D_{0} \cdot R_{C 1}+R / / R_{C 2}\right] & \frac{1}{L_{2}} & -\frac{1}{L_{2}} \frac{R}{R+R_{C 2}} \\
\frac{1}{C_{1}} & -\frac{1}{C_{1}} & 0 & 0 \\
-D_{0} \frac{R}{C_{2}\left(R+R_{C 2}\right)} & \frac{1}{C_{2}} \frac{R}{R+R_{C 2}} & 0 & -\frac{1}{C_{2}\left(R+R_{C 2}\right)}
\end{array}\right]
\end{aligned}
$$

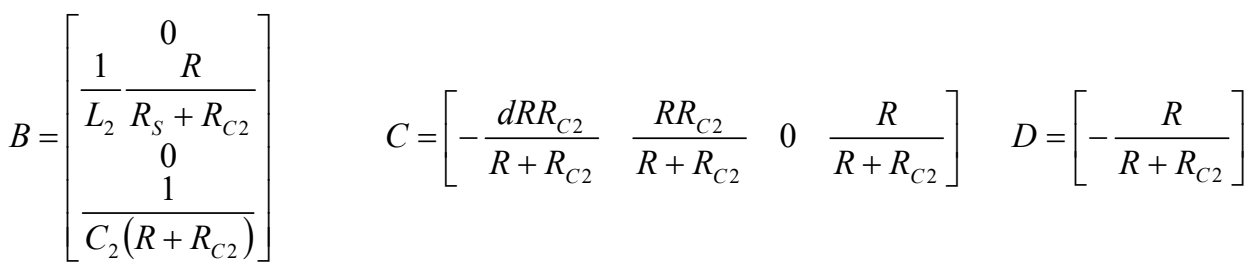




\section{Creative Commons Attribution License 4.0} (Attribution 4.0 International, CC BY 4.0)

This article is published under the terms of the Creative Commons Attribution License 4.0

https://creativecommons.org/licenses/by/4.0/deed.en_US 